\title{
STABLE DISCRETIZATION OF MAGNETOHYDRODYNAMICS IN BOUNDED DOMAINS*
}

\author{
JIAN-GUO LIU† AND ROBERT L. PEGO ${ }^{\ddagger}$
}

Dedicated to the sixtieth birthday of Professor Andrew Majda

\begin{abstract}
We study a semi-implicit time-difference scheme for magnetohydrodynamics of a viscous and resistive incompressible fluid in a bounded smooth domain with a perfectly conducting boundary. In the scheme, the velocity and magnetic fields are updated by solving simple Helmholtz equations. Pressure is treated explicitly in time, by solving Poisson equations corresponding to a recently developed formula for the Navier-Stokes pressure involving the commutator of Laplacian and Leray projection operators. We prove stability of the time-difference scheme, and deduce a local-time wellposedness theorem for MHD dynamics extended to ignore the divergence-free constraint on velocity and magnetic fields. These fields are divergence-free for all later time if they are initially so.
\end{abstract}

Key words. Time-dependent incompressible viscous flow, Stokes pressure, Leray projection, projection method, pressure Poisson equation.

AMS subject classifications. 76W05, 76D03.

\section{Introduction}

The equations of magnetohydrodynamics (MHD) for incompressible, viscous and resistive, electrically conducting fluid flow take the form [9]

$$
\begin{aligned}
\partial_{t} \mathbf{u}+\mathbf{u} \cdot \nabla \mathbf{u}+\nabla p & =\nu \Delta \mathbf{u}+\alpha(\nabla \times \mathbf{b}) \times \mathbf{b}+\mathbf{f}, \\
\partial_{t} \mathbf{b}+\nabla \times(\mathbf{b} \times \mathbf{u}) & =\eta \Delta \mathbf{b}, \\
\nabla \cdot \mathbf{u} & =0 \\
\nabla \cdot \mathbf{b} & =0 .
\end{aligned}
$$

Examples of such fluids include plasmas, liquid metals (mercury, liquid sodium), and salt water. Here $\mathbf{u}$ is the fluid velocity, $p$ is the pressure, $\mathbf{f}$ is the external force, and $\mathbf{b}$ is the magnetic field (more properly, flux density or induction). The coefficients $\nu, \eta$, and $\alpha$ are assumed to be fixed positive constants and represent, respectively, the kinematic viscosity, the magnetic diffusivity, and $\alpha=1 /(4 \pi \mu \rho)$ where $\mu$ is the magnetic permeability and $\rho$ is the fluid density.

In general, the magnetic field penetrates the boundary and interacts with the outside environment. For simplicity and in order to focus on the main issues of concern here, we consider MHD in a perfectly conducting container. This confines the magnetic field inside and decouples it from the exterior. We assume the flow is contained in a bounded and connected domain $\Omega \subset \mathbb{R}^{N}(N=2$ or 3$)$ with smooth boundary $\Gamma=\partial \Omega$. (When $N=2$ the velocity and magnetic fields take values in $\mathbb{R}^{2}$, considered as embedded in $\mathbb{R}^{3}$ with zero third component.) We specify the velocity on $\Gamma$, with no sources or sinks of fluid, requiring

$$
\mathbf{u}=\mathbf{g}, \quad \mathbf{n} \cdot \mathbf{g}=0 \quad \text { on } \Gamma .
$$

\footnotetext{
*Received: September 30, 2008; accepted (in revised version): January 9, 2009

${ }^{\dagger}$ Department of Mathematics and Department of Physics, Duke University, Durham, NC 27708, (jian-guo.liu@duke.edu).

${ }^{\ddagger}$ Mathematical Sciences and Center for Nonlinear Analysis, Carnegie Mellon University, Pittsburgh, PA 15213, (rpego@cmu.edu).
} 
We refer to this as a no-flow boundary condition. When $\mathbf{g} \equiv 0$, we refer to it as a no-slip boundary condition. Requiring the container be perfectly conducting means requiring

$$
\mathbf{n} \times \mathbf{e}=0, \quad \mathbf{n} \cdot \mathbf{b}=0 \quad \text { on } \Gamma,
$$

where $\mathbf{e}=c^{-1}(\mathbf{b} \times \mathbf{u}+\eta \nabla \times \mathbf{b})$ is the electric field ( $c$ is the speed of light). If we assume $\mathbf{n} \cdot \mathbf{b}=0$ on $\Gamma$, then the boundary condition $\mathbf{n} \times \mathbf{e}=0$ becomes

$$
\mathbf{n} \times(\nabla \times \mathbf{b})=-\frac{1}{\eta}(\mathbf{n} \cdot \mathbf{g}) \mathbf{b} \quad \text { on } \Gamma,
$$

which is similar to the Navier slip boundary condition. Because we impose the no flow boundary condition $\mathbf{n} \cdot \mathbf{g}=0$, the boundary conditions in (1.6) for the magnetic field take the form

$$
\mathbf{n} \times(\nabla \times \mathbf{b})=0, \quad \mathbf{n} \cdot \mathbf{b}=0 \quad \text { on } \Gamma .
$$

Our aim is to study the stability of discretization schemes for these MHD equations in bounded domains, supplemented with initial conditions of the form

$$
\mathbf{u}(\cdot, 0)=\mathbf{u}_{\text {in }}, \quad \mathbf{b}(\cdot, 0)=\mathbf{b}_{\text {in }} \quad \text { in } \Omega .
$$

Particular attention must be paid to the constraint that the magnetic field and velocity must be divergence-free. We will demonstrate, however, that updating the velocity and magnetic field can be based on simple Helmholtz equations, and the pressure can be separately computed by Poisson equations. The analysis is based on a commutator formula and estimates for pressure that derive from our work on incompressible Navier-Stokes equations [11, 12, 13]. These estimates show that the pressure gradient is strictly dominated by the viscosity term in $L^{2}$-norm at leading order. One aim of the present work is to demonstrate the utility of these estimates for studying problems coupling incompressible viscous flow to more complicated physics.

From this stability analysis we obtain a local-time well-posedness theorem for strong solutions of the MHD equations (1.1), (1.2) with boundary conditions (1.5), (1.8) but without the divergence-free constraints (1.3)-(1.4); pressure is determined as described in the next section below. The velocity and magnetic fields will be divergence-free for all time if they are divergence-free at the initial time (they satisfy diffusion equations with no-flux boundary conditions). We will show that these unconstrained MHD equations have a locally unique strong solution with the regularity

$$
\begin{aligned}
& \mathbf{u}, \mathbf{b} \in L^{2}\left(0, T ; H^{2}\left(\Omega, \mathbb{R}^{N}\right)\right) \cap H^{1}\left(0, T ; L^{2}\left(\Omega, \mathbb{R}^{N}\right)\right), \\
& \nabla p \in L^{2}\left(0, T ; L^{2}\left(\Omega, \mathbb{R}^{N}\right)\right),
\end{aligned}
$$

provided that

$$
\begin{aligned}
& \mathbf{u}_{\text {in }}, \mathbf{b}_{\text {in }} \in H^{1}\left(\Omega, \mathbb{R}^{N}\right), \\
& \mathbf{f} \in L^{2}\left(0, T ; L^{2}\left(\Omega, \mathbb{R}^{N}\right)\right), \\
& \mathbf{g} \in H_{g}:=H^{3 / 4}\left(0, T ; L^{2}\left(\Gamma, \mathbb{R}^{N}\right)\right) \cap L^{2}\left(0, T ; H^{3 / 2}\left(\Gamma, \mathbb{R}^{N}\right)\right),
\end{aligned}
$$

and provided that the appropriate compatibility conditions hold:

$$
\mathbf{n} \cdot \mathbf{u}_{\text {in }}=0, \quad \mathbf{n} \cdot \mathbf{b}_{\text {in }}=0, \quad \mathbf{n} \cdot \mathbf{g}=0, \quad \mathbf{u}_{\text {in }}=\mathbf{g}(\cdot, 0) \quad \text { on } \Gamma .
$$


The space $H_{g}$ is the space of boundary traces of functions in (1.10), see [14].

We remark that in the case of two dimensions $(N=2)$ and for divergence-free fields, global existence results are classical, see [4, 15]. However, we have not managed to extend these to the present case without divergence constraints.

We anticipate that this unconstrained formulation will serve as a starting point for the development of more accurate and flexible numerical schemes for MHD, much as the well-posedness theory for the Navier-Stokes equations in [11] served as a basis for the significantly improved numerical methods described in $[12,13]$.

\section{Preliminaries}

2.1. The Laplace-Leray commutator. Recall that an arbitrary squareintegrable velocity field $\mathbf{u}$ has a unique Helmholtz decomposition

$$
\mathbf{u}=\mathbf{v}+\nabla \phi
$$

where $\mathbf{v}$ is $L^{2}$-orthogonal to all square-integrable gradients: $\int_{\Omega} \mathbf{v} \cdot \nabla q=0$ for all $q$ smooth enough. Then $\mathbf{v}$ is divergence-free and at the boundary has a vanishing component in the direction of the outward unit normal $\mathbf{n}$ :

$$
\nabla \cdot \mathbf{v}=0 \quad \text { in } \Omega, \quad \mathbf{n} \cdot \mathbf{v}=0 \quad \text { on } \Gamma .
$$

We write $\mathbf{v}=\mathcal{P} \mathbf{u}$, defining the Leray-Helmholtz projection operator $\mathcal{P}$, and write $\phi=\mathcal{Q} \mathbf{u}$ to denote the zero-mean potential field in $(2.1)$. That is, $\nabla \phi=(I-\mathcal{P}) \mathbf{u}$. Then since $\Delta \phi=\nabla \cdot \mathbf{u}$, we find $\Delta(I-\mathcal{P}) \mathbf{u}=\Delta \nabla \phi=\nabla \Delta \phi=\nabla \nabla \cdot \mathbf{u}$, and from this, the fact $\mathcal{P} \nabla=0$, and the vector identity

$$
\nabla \times \nabla \times \mathbf{u}=-\Delta \mathbf{u}+\nabla \nabla \cdot \mathbf{u}
$$

one immediately obtains the following identities described in [11]:

$$
\begin{aligned}
\Delta \mathcal{P} \mathbf{u} & =(\Delta-\nabla \nabla \cdot) \mathbf{u}=-\nabla \times \nabla \times \mathbf{u}, \\
(\Delta \mathcal{P}-\mathcal{P} \Delta) \mathbf{u} & =(I-\mathcal{P})(\Delta-\nabla \nabla \cdot) \mathbf{u}=-(I-\mathcal{P})(\nabla \times \nabla \times \mathbf{u}) .
\end{aligned}
$$

In (2.5) we require $\mathbf{u} \in H^{2}\left(\Omega, \mathbb{R}^{N}\right)$. Then we see that the commutator of the Laplacian and Leray projection operators is the gradient of a potential field $p_{\mathrm{S}}(\mathbf{u})$ satisfying

$$
p_{\mathrm{S}}(\mathbf{u})=\mathcal{Q}(\Delta-\nabla \nabla \cdot) \mathbf{u}, \quad \nabla p_{\mathrm{S}}(\mathbf{u})=(\Delta \mathcal{P}-\mathcal{P} \Delta) \mathbf{u}
$$

From (2.6) it follows that $p_{\mathrm{S}}(\mathbf{u})$ is the unique zero-mean solution of the boundary value problem

$$
\Delta p_{\mathrm{S}}(\mathbf{u})=0 \quad \text { in } \Omega, \quad \mathbf{n} \cdot \nabla p_{\mathrm{S}}(\mathbf{u})=\mathbf{n} \cdot(\Delta-\nabla \nabla \cdot) \mathbf{u} \quad \text { on } \Gamma .
$$

(Since $(\Delta-\nabla \nabla \cdot) \mathbf{u}$ has zero divergence, the boundary condition holds in $H^{-1 / 2}(\Gamma)$, due to a standard trace theorem.) The following estimate from [11] will play a key role in our stability analysis.

TheOrem 2.1. Suppose $\Omega$ is a bounded domain with $C^{3}$ boundary, and $\varepsilon>0$. Then there is a constant $C$ such that for all $\mathbf{u} \in H^{2} \cap H_{0}^{1}\left(\Omega, \mathbb{R}^{N}\right)$,

$$
\int_{\Omega}\left|\nabla p_{S}(\mathbf{u})\right|^{2} \leq\left(\frac{1}{2}+\varepsilon\right) \int_{\Omega}|\Delta \mathbf{u}|^{2}+C \int_{\Omega}|\nabla \mathbf{u}|^{2} .
$$

If $\Omega$ is replaced by a half-space, the estimate (2.8) holds with $\varepsilon=C=0$ and is sharp; see [11]. 
2.2. Formula for pressure. Suppose now that $\mathbf{u}$ is a (sufficiently regular) solution of (1.1) satisfying (1.3) and (1.5). Then $\mathbf{u}=\mathcal{P} \mathbf{u}$. We apply $\mathcal{P}$ to (1.2), while collecting together the nonlinear and forcing terms in (1.1) to write

$$
\mathbf{f}_{\mathrm{tot}}=-\mathbf{u} \cdot \nabla \mathbf{u}+\alpha(\nabla \times \mathbf{b}) \times \mathbf{b}+\mathbf{f} .
$$

If we use (2.6) to say $\mathcal{P} \Delta \mathbf{u}=\Delta \mathcal{P} \mathbf{u}-\nabla p_{\mathrm{S}}(\mathbf{u})$, since $\mathcal{P} \nabla p=0$ we find

$$
\partial_{t} \mathbf{u}+\nu \nabla p_{\mathrm{S}}(\mathbf{u})=\nu \Delta \mathbf{u}+\mathcal{P} \mathbf{f}_{\mathrm{tot}} .
$$

Since $\mathcal{P}=I-\nabla \mathcal{Q}$, comparing (2.10) with (1.2) shows that necessarily (up to constants)

$$
p=\nu p_{\mathrm{S}}(\mathbf{u})+\mathcal{Q} \mathbf{f}_{\mathrm{tot}} .
$$

This formula expresses the pressure directly in terms of the current velocity, magnetic, and forcing fields. We refer to $p_{\mathrm{S}}(\mathbf{u})$ as the Stokes pressure since the other terms vanish when forcing and nonlinear terms are absent.

For numerical computation of this pressure by finite element methods, it is best to base discretization on the following weak-form characterization that involves only first derivatives: For all test functions $\psi$ with square-integrable gradient,

$$
\int_{\Omega} \nabla p \cdot \nabla \psi=\int_{\Gamma} \nu(\nabla \times \mathbf{u}) \cdot(\mathbf{n} \times \nabla \psi)+\int_{\Omega} \mathbf{f}_{\mathrm{tot}} \cdot \nabla \psi .
$$

This means that for sufficiently regular data, $p$ is determined by the boundary value problem

$$
\begin{aligned}
\Delta p & =\nabla \cdot \mathbf{f}_{\mathrm{tot}} \quad \text { in } \Omega, \\
\mathbf{n} \cdot \nabla p & =-\mathbf{n} \cdot(\nu \nabla \times \nabla \times \mathbf{u})+\mathbf{n} \cdot \mathbf{f}_{\mathrm{tot}} \quad \text { on } \Gamma .
\end{aligned}
$$

2.3. Div-curl norms and calculus inequalities. Below, we let $\langle f, g\rangle_{\Omega}=$ $\int_{\Omega} f g$ denote the $L^{2}$ inner product of functions $f$ and $g$ in $\Omega$, and let $\|\cdot\|_{\Omega}$ denote the corresponding norm in $L^{2}(\Omega)$. We drop the subscript on the inner product and norm when the domain of integration is understood in context.

We let

$$
V:=\left\{\mathbf{v} \in L^{2}\left(\Omega, \mathbb{R}^{N}\right): \nabla \cdot \mathbf{v} \in L^{2}(\Omega), \nabla \times \mathbf{v} \in L^{2}\left(\Omega, \mathbb{R}^{3}\right),\left.\mathbf{n} \cdot \mathbf{v}\right|_{\Gamma} \in H^{1 / 2}(\Gamma)\right\}
$$

denote the space of square-integrable vector fields on $\Omega$ with square-integrable divergence and curl, with normal component at the boundary in the space of traces of $H^{1}$ functions. On $V$ we use the norm

$$
\|\mathbf{v}\|_{V}^{2}=\|\mathbf{v}\|^{2}+\|\nabla \cdot \mathbf{v}\|^{2}+\|\nabla \times \mathbf{v}\|^{2}+\|\mathbf{n} \cdot \mathbf{v}\|_{H^{1 / 2}(\Gamma)}^{2}
$$

From [3, Prop. 6, p.235], it follows $V=H^{1}\left(\Omega, \mathbb{R}^{N}\right)$, and the norm above is equivalent to the usual $H^{1}$ norm.

Next we introduce $V^{\Delta}:=\left\{\mathbf{v} \in V: \Delta \mathbf{v} \in L^{2}\left(\Omega, \mathbb{R}^{N}\right)\right\}$, and the norm

$$
\|\mathbf{v}\|_{W}^{2}=\|\mathbf{v}\|_{V}^{2}+\|\Delta \mathbf{v}\|^{2} \text {. }
$$

The space of smooth functions $C^{\infty}\left(\Omega, \mathbb{R}^{N}\right)$ is dense in $V^{\Delta}$ with this norm. (This is not difficult to prove by a standard technique, see [1, Thm. 3.18].) We claim that the map

$$
\mathbf{v} \mapsto \beta \mathbf{v}=-\mathbf{n} \times(\nabla \times \mathbf{v})+\mathbf{n}(\nabla \cdot \mathbf{v})
$$


extends to a bounded map from $V^{\Delta}$ to $H^{-3 / 2}\left(\Gamma, \mathbb{R}^{N}\right)$. To see this, observe that and whenever $\mathbf{v}, \mathbf{w} \in V^{\Delta}$ are smooth, we have the Green's formula

$$
\langle\Delta \mathbf{v}, \mathbf{w}\rangle_{\Omega}-\langle\mathbf{v}, \Delta \mathbf{w}\rangle_{\Omega}=\langle\beta \mathbf{v}, \mathbf{w}\rangle_{\Gamma}-\langle\mathbf{v}, \beta \mathbf{w}\rangle_{\Gamma} .
$$

By standard extension theorems, there is a bounded map $\mathbf{g} \mapsto \mathbf{w}$ from $H^{3 / 2}\left(\Gamma, \mathbb{R}^{N}\right)$ to $H^{2}\left(\Omega, \mathbb{R}^{N}\right)$ such that $\left.\mathbf{w}\right|_{\Gamma}=\mathbf{g}$. Then (2.18) implies that the map $\beta$ extends as claimed, and (2.18) holds for all $\mathbf{v} \in V^{\Delta}$ and $\mathbf{w} \in H^{2}\left(\Omega, \mathbb{R}^{N}\right)$.

For later use, we introduce the space

$$
W:=\left\{\mathbf{v} \in V^{\Delta}: \mathbf{n} \cdot \mathbf{v}=0, \mathbf{n} \times(\nabla \times \mathbf{v})=0 \text { on } \Gamma\right\},
$$

with norm given by (2.17). By the result of Lemma 2.2 below, we have that $W \subset$ $H^{2}\left(\Omega, \mathbb{R}^{N}\right)$ and the norm in $(2.17)$ is equivalent to usual the $H^{2}$ norm on $W$. It is then easy to show that if $\mathbf{v} \in W$, then

$$
\|\Delta \mathbf{v}\|^{2}=\|\nabla \times \nabla \times \mathbf{v}\|^{2}+\|\nabla \nabla \cdot \mathbf{v}\|^{2} .
$$

To establish the solvability of the time-discrete scheme that we will study, we use the following lemma.

LEMmA 2.2. Let $\lambda>0$ and assume $\Omega \subset \mathbb{R}^{N}$ is a bounded domain with smooth boundary, $N=2$ or 3 . Then for any $\mathbf{f} \in L^{2}\left(\Omega, \mathbb{R}^{N}\right)$, there is a unique $\mathbf{v} \in V \cap H^{2}\left(\Omega, \mathbb{R}^{N}\right)$ such that

$$
\begin{array}{rlr}
\lambda \mathbf{v}-\Delta \mathbf{v}=\mathbf{f} & \text { in } \Omega, \\
\mathbf{n} \cdot \mathbf{v}=0, \quad \mathbf{n} \times(\nabla \times \mathbf{v})=0 & \text { on } \Gamma .
\end{array}
$$

Further, there is a constant $C \geq 0$ independent of $\mathbf{f}$ such that

$$
\|\mathbf{v}\|_{H^{2}(\Omega)} \leq C\|\mathbf{f}\|_{L^{2}(\Omega)} .
$$

Proof. Formally testing (2.20) by $\mathbf{w}$ and integrating by parts, we arrive at the following weak form of (2.20)-(2.21): Let $V_{0}=\{\mathbf{v} \in V: \mathbf{n} \cdot \mathbf{v}=0$ on $\Gamma\}$. Find $\mathbf{v} \in V_{0}$ such that for all $\mathbf{w} \in V_{0}$,

$$
\lambda\langle\mathbf{v}, \mathbf{w}\rangle+\langle\nabla \cdot \mathbf{v}, \nabla \cdot \mathbf{w}\rangle+\langle\nabla \times \mathbf{v}, \nabla \times \mathbf{w}\rangle=\langle\mathbf{f}, \mathbf{w}\rangle .
$$

Using the norm equivalence referred to above, existence, uniqueness, and boundedness in $H^{1}$ of the solution to this problem is a simple consequence of the Lax-Milgram theorem. Taking $\mathbf{w}$ as a smooth test function, we find (2.20) holds in the sense of distributions and $\Delta \mathbf{v} \in L^{2}$. Taking $\mathbf{w}$ smooth with $\mathbf{n} \cdot \mathbf{w}=0$ on $\Gamma$, from (2.23) we infer then that

$$
\begin{aligned}
\langle\mathbf{f}, \mathbf{w}\rangle & =\langle\mathbf{v}, \lambda \mathbf{w}-\Delta \mathbf{w}\rangle+\langle\mathbf{v}, \mathbf{n} \times(\nabla \times \mathbf{w})\rangle_{\Gamma} \\
& =\langle\lambda \mathbf{v}-\Delta \mathbf{v}, \mathbf{w}\rangle+\langle\mathbf{n} \times(\nabla \times \mathbf{v}), \mathbf{w}\rangle_{\Gamma},
\end{aligned}
$$

by invoking (2.18), and it follows $\mathbf{n} \times(\nabla \times \mathbf{v})=0$ in $H^{-3 / 2}\left(\Gamma, \mathbb{R}^{N}\right)$. It then follows directly from a regularity result of Georgescu [7, Thm. 3.2.3], that $\mathbf{v} \in H^{2}\left(\Omega, \mathbb{R}^{N}\right)$, and the estimate (2.22) is a consequence of the inverse mapping theorem. 

$[10$

For estimating nonlinear terms, we will make use of Ladyzhenskaya's inequalities

$$
\begin{array}{ll}
\int_{\mathbb{R}^{N}} g^{4} \leq 2\left(\int_{\mathbb{R}^{N}} g^{2}\right)\left(\int_{\mathbb{R}^{N}}|\nabla g|^{2}\right) & (N=2), \\
\int_{\mathbb{R}^{N}} g^{4} \leq 4\left(\int_{\mathbb{R}^{N}} g^{2}\right)^{1 / 2}\left(\int_{\mathbb{R}^{N}}|\nabla g|^{2}\right)^{3 / 2} & (N=3),
\end{array}
$$

valid for $g \in H^{1}\left(\mathbb{R}^{N}\right)$ with $N=2$ and 3 respectively, in combination with the fact that the standard bounded extension operator $H^{1}(\Omega) \rightarrow H^{1}\left(\mathbb{R}^{N}\right)$ is also bounded in $L^{2}$ norm, to deduce that for all $g \in H^{1}(\Omega)$,

$$
\begin{array}{ll}
\|g\|_{L^{4}}^{2} \leq C\|g\|_{L^{2}}\|g\|_{H^{1}} & (N=2), \\
\|g\|_{L^{3}}^{2} \leq\|g\|_{L^{2}}^{2 / 3}\|g\|_{L^{4}}^{4 / 3} \leq C\|g\|_{L^{2}}\|g\|_{H^{1}} & (N=3) .
\end{array}
$$

\section{Stability analysis for a time-discrete scheme}

3.1. Unconstrained MHD system. The traditional way to regard the pressure in (1.1) is that it is to be determined so that the divergence-free condition (1.3) holds. Our aim, however, is to show that the MHD system (1.1)-(1.2), with the boundary conditions (1.5) and (1.8), is stably approximated by discretization and indeed becomes well posed if the pressure formula (2.11) is used to determine pressure, regardless of whether velocity and magnetic fields are initially divergence-free or not.

The divergences of the velocity and magnetic fields will turn out to satisfy diffusion equations with no-flux boundary conditions. Let us describe how this works formally. For the velocity, let $\phi$ be an arbitrary smooth test function and note that

$$
\left\langle\nabla p_{\mathrm{S}}(\mathbf{u}), \nabla \phi\right\rangle=\langle(\Delta-\nabla \nabla \cdot) \mathbf{u}, \nabla \phi\rangle
$$

by (2.6) and (2.5). Then by testing (2.10) with $\nabla \phi$, we find that

$$
\left\langle\partial_{t} \mathbf{u}, \nabla \phi\right\rangle=\langle\nu \nabla \nabla \cdot \mathbf{u}, \nabla \phi\rangle
$$

and this is the weak form of the equations

$$
\partial_{t}(\nabla \cdot \mathbf{u})=\nu \Delta(\nabla \cdot \mathbf{u}) \quad \text { in } \Omega, \quad \mathbf{n} \cdot \nabla(\nabla \cdot \mathbf{u})=0 \quad \text { on } \Gamma .
$$

For the magnetic field, observe that for any smooth $\phi$ we have

$$
\begin{array}{r}
\langle\nabla \times \nabla \times \mathbf{b}, \nabla \phi\rangle=\langle\mathbf{n} \times \nabla \times \mathbf{b}, \nabla \phi\rangle_{\Gamma}=0, \\
\langle\nabla \times(\mathbf{b} \times \mathbf{u}), \nabla \phi\rangle=\langle\mathbf{n} \times(\mathbf{b} \times \mathbf{u}), \nabla \phi\rangle_{\Gamma}=0,
\end{array}
$$

since $\mathbf{n} \times(\mathbf{b} \times \mathbf{u})=(\mathbf{n} \cdot \mathbf{u}) \mathbf{b}-(\mathbf{n} \cdot \mathbf{b}) \mathbf{u}=0$ on $\Gamma$. Thus, testing (1.2) with $\nabla \phi$ and using (2.3) we find

$$
\left\langle\partial_{t} \mathbf{b}, \nabla \phi\right\rangle=\langle\eta \nabla \nabla \cdot \mathbf{b}, \nabla \phi\rangle
$$

and this is the weak form of

$$
\partial_{t}(\nabla \cdot \mathbf{b})=\eta \Delta(\nabla \cdot \mathbf{b}) \quad \text { in } \Omega, \quad \mathbf{n} \cdot \nabla(\nabla \cdot \mathbf{b})=0 \quad \text { on } \Gamma .
$$


3.2. Time discretization. Our main aim is to study the following timediscretization scheme, implicit only in the viscosity and resistivity terms and explicit in the pressure and nonlinear terms.

We assume $\mathbf{u}_{\text {in }}, \mathbf{b}_{\text {in }} \in V$, and for some given $T>0, \mathbf{f} \in L^{2}\left(0, T ; L^{2}\left(\Omega, \mathbb{R}^{N}\right)\right)$ and $\mathbf{g} \in H_{g}$ with $\mathbf{n} \cdot \mathbf{g}=0$ on $\Gamma$. We take $\mathbf{u}^{0}, \mathbf{b}^{0} \in W=V \cap H^{2}\left(\Omega, \mathbb{R}^{N}\right)$ to approximate $\mathbf{u}_{\text {in }}$ and $\mathbf{b}_{\text {in }}$ in $H^{1}\left(\Omega, \mathbb{R}^{N}\right)$, respectively, and set

$$
\mathbf{f}^{n}=\frac{1}{\Delta t} \int_{n \Delta t}^{(n+1) \Delta t} \mathbf{f}(t) d t, \quad \mathbf{g}^{n}=\frac{1}{\Delta t} \int_{n \Delta t}^{(n+1) \Delta t} \mathbf{g}(t) d t .
$$

We consider the following time-discrete scheme: Find $\mathbf{u}^{n+1}, \mathbf{b}^{n+1} \in H^{2}\left(\Omega, \mathbb{R}^{N}\right)(n \geq 0)$ such that

$$
\begin{aligned}
& \frac{\mathbf{u}^{n+1}-\mathbf{u}^{n}}{\Delta t}-\nu \Delta \mathbf{u}^{n+1}=-\nabla p^{n}+\mathbf{f}_{\text {tot }}^{n} \quad \text { in } \Omega, \\
& \frac{\mathbf{b}^{n+1}-\mathbf{b}^{n}}{\Delta t}-\eta \Delta \mathbf{b}^{n+1}=-\nabla \times\left(\mathbf{b}^{n} \times \mathbf{u}^{n}\right) \quad \text { in } \Omega, \\
& \mathbf{u}^{n+1}=\mathbf{g}^{n+1}, \quad \mathbf{n} \times\left(\nabla \times \mathbf{b}^{n+1}\right)=0, \quad \mathbf{n} \cdot \mathbf{b}^{n+1}=0 \quad \text { on } \Gamma,
\end{aligned}
$$

where

$$
\mathbf{f}_{\text {tot }}^{n}=-\mathbf{u}^{n} \cdot \nabla \mathbf{u}^{n}+\alpha\left(\nabla \times \mathbf{b}^{n}\right) \times \mathbf{b}^{n}+\mathbf{f}^{n},
$$

and where we determine $\nabla p^{n}$ from a weak-form pressure Poisson equation corresponding to (2.12), requiring

$$
\left\langle\nabla p^{n}, \nabla \psi\right\rangle=\nu\left\langle\nabla \times \mathbf{u}^{n}, \mathbf{n} \times \nabla \psi\right\rangle_{\Gamma}+\left\langle\mathbf{f}_{\mathrm{tot}}^{n}, \nabla \psi\right\rangle \quad \forall \psi \in H^{1}(\Omega) .
$$

This means that

$$
-\nabla p^{n}+\mathbf{f}_{\mathrm{tot}}^{n}=-\nu \nabla p_{\mathrm{S}}\left(\mathbf{u}^{n}\right)+\mathcal{P} \mathbf{f}_{\mathrm{tot}}^{n} .
$$

The unique solvability of (3.10) with the boundary conditions in (3.11) is a consequence of Lemma 2.2.

3.3. Stability analysis for no-slip boundary conditions. For simplicity, at first we consider no-slip boundary conditions, taking $\mathbf{g}=0$. Our goal in this section is to prove the following stability estimate for the time-discrete scheme in (3.9)-(3.13).

THEOREM 3.1. Let $\Omega$ be a bounded domain in $\mathbb{R}^{N}$ ( $N=2$ or 3 ) with smooth boundary, and let $\nu, \eta, M_{0}>0$. Then there exist $T_{*}, C_{3}>0$ such that, if $\mathbf{u}^{0} \in H_{0}^{1} \cap H^{2}\left(\Omega, \mathbb{R}^{N}\right)$, $\mathbf{b}^{0} \in W, \mathbf{g}=0$, and $\mathbf{f} \in L^{2}\left(0, T ; L^{2}\left(\Omega, \mathbb{R}^{N}\right)\right)$ for some $T \in\left(0, T_{*}\right]$, with

$$
\left\|\mathbf{b}^{0}\right\|_{H^{1}}^{2}+\left\|\nabla \mathbf{u}^{0}\right\|^{2}+\eta \Delta t\left\|\Delta \mathbf{b}^{0}\right\|^{2}+\nu \Delta t\left\|\Delta \mathbf{u}^{0}\right\|^{2}+\int_{0}^{T}\|\mathbf{f}(t)\|^{2} d t \leq M_{0},
$$

then whenever $2 \eta \Delta t \leq 1$ and $0<(n+1) \Delta t \leq T$, the solution to the time-discrete scheme (3.9)-(3.13) satisfies

$$
\begin{aligned}
& \sup _{0 \leq k \leq n}\left(\left\|\mathbf{b}^{k}\right\|_{H^{1}}^{2}+\left\|\nabla \mathbf{u}^{k}\right\|^{2}\right)+\sum_{k=0}^{n}\left(\left\|\Delta \mathbf{b}^{k}\right\|^{2}+\left\|\Delta \mathbf{u}^{k}\right\|^{2}\right) \Delta t \leq C_{3}, \\
& \left.\sum_{k=0}^{n-1}\left(\left\|\nabla \times\left(\mathbf{b}^{k} \times \mathbf{u}^{k}\right)\right\|^{2}+\|\left(\nabla \times \mathbf{b}^{k}\right) \times \mathbf{b}^{k}\right)\left\|^{2}+\right\| \mathbf{u}^{k} \cdot \nabla \mathbf{u}^{k} \|^{2}\right) \Delta t \leq C_{3} . \\
& \sum_{k=0}^{n-1}\left(\left\|\frac{\mathbf{b}^{k+1}-\mathbf{b}^{k}}{\Delta t}\right\|^{2}+\left\|\frac{\mathbf{u}^{k+1}-\mathbf{u}^{k}}{\Delta t}\right\|^{2}\right) \Delta t \leq C_{3} .
\end{aligned}
$$


Proof.

1. Testing $\mathbf{b}^{n+1}-\Delta \mathbf{b}^{n+1}$ against the various terms in (3.10) and using the boundary conditions, we find

$$
\begin{aligned}
\left\langle\mathbf{b}^{n+1}-\Delta \mathbf{b}^{n+1}, \frac{\mathbf{b}^{n+1}-\mathbf{b}^{n}}{\Delta t}\right\rangle= & \frac{1}{2 \Delta t}\left(\left\|\mathbf{b}^{n+1}\right\|_{V}^{2}-\left\|\mathbf{b}^{n}\right\|_{V}^{2}+\left\|\mathbf{b}^{n+1}-\mathbf{b}^{n}\right\|_{V}^{2}\right), \\
\left\langle\mathbf{b}^{n+1}-\Delta \mathbf{b}^{n+1},-\eta \Delta \mathbf{b}^{n+1}\right\rangle= & \eta\left\|\Delta \mathbf{b}^{n+1}\right\|^{2}+\eta\left\|\nabla \cdot \mathbf{b}^{n+1}\right\|^{2}+\eta\left\|\nabla \times \mathbf{b}^{n+1}\right\|^{2}, \\
\left\langle\mathbf{b}^{n+1}-\Delta \mathbf{b}^{n+1}, \nabla \times\left(\mathbf{b}^{n} \times \mathbf{u}^{n}\right)\right\rangle \leq & \frac{\eta}{2}\left(\left\|\Delta \mathbf{b}^{n+1}\right\|^{2}+\left\|\nabla \times \mathbf{b}^{n+1}\right\|^{2}\right) \\
& +\frac{1}{2 \eta}\left\|\nabla \times\left(\mathbf{b}^{n} \times \mathbf{u}^{n}\right)\right\|^{2}+\frac{1}{2 \eta}\left\|\mathbf{b}^{n} \times \mathbf{u}^{n}\right\|^{2} .
\end{aligned}
$$

Combining these estimates with (3.10) one has

$$
\begin{aligned}
& \frac{\left\|\mathbf{b}^{n+1}\right\|_{V}^{2}-\left\|\mathbf{b}^{n}\right\|_{V}^{2}+\left\|\mathbf{b}^{n+1}-\mathbf{b}^{n}\right\|_{V}^{2}}{\Delta t}+\eta\left(\left\|\Delta \mathbf{b}^{n+1}\right\|^{2}+\left\|\nabla \cdot \mathbf{b}^{n+1}\right\|^{2}+\left\|\nabla \times \mathbf{b}^{n+1}\right\|^{2}\right) \\
\leq & \frac{1}{\eta}\left\|\nabla \times\left(\mathbf{b}^{n} \times \mathbf{u}^{n}\right)\right\|^{2}+\frac{1}{\eta}\left\|\mathbf{b}^{n} \times \mathbf{u}^{n}\right\|^{2} .
\end{aligned}
$$

This gives

$$
\begin{aligned}
& \frac{\left\|\mathbf{b}^{n+1}\right\|_{V}^{2}-\left\|\mathbf{b}^{n}\right\|_{V}^{2}+\left\|\mathbf{b}^{n+1}-\mathbf{b}^{n}\right\|_{V}^{2}}{\Delta t}+\eta\left(\left\|\mathbf{b}^{n+1}\right\|_{W}^{2}-\left\|\mathbf{b}^{n}\right\|_{W}^{2}\right)+\eta\left\|\mathbf{b}^{n}\right\|_{W}^{2} \\
\leq & \frac{1}{\eta}\left\|\nabla \times\left(\mathbf{b}^{n} \times \mathbf{u}^{n}\right)\right\|^{2}+\frac{1}{\eta}\left\|\mathbf{b}^{n} \times \mathbf{u}^{n}\right\|^{2}+\eta\left\|\mathbf{b}^{n+1}\right\|^{2} .
\end{aligned}
$$

We assume $2 \eta \Delta t \leq 1$, thus

$$
\eta\left\|\mathbf{b}^{n+1}\right\|^{2} \leq 2 \eta\left\|\mathbf{b}^{n+1}-\mathbf{b}^{n}\right\|^{2}+2 \eta\left\|\mathbf{b}^{n}\right\|^{2} \leq \frac{\left\|\mathbf{b}^{n+1}-\mathbf{b}^{n}\right\|_{V}^{2}}{\Delta t}+2 \eta\left\|\mathbf{b}^{n}\right\|^{2},
$$

and hence

$$
\begin{aligned}
& \frac{\left\|\mathbf{b}^{n+1}\right\|_{V}^{2}-\left\|\mathbf{b}^{n}\right\|_{V}^{2}}{\Delta t}+\eta\left(\left\|\mathbf{b}^{n+1}\right\|_{W}^{2}-\left\|\mathbf{b}^{n}\right\|_{W}^{2}\right)+\eta\left\|\mathbf{b}^{n}\right\|_{W}^{2} \\
\leq & \frac{1}{\eta}\left\|\nabla \times\left(\mathbf{b}^{n} \times \mathbf{u}^{n}\right)\right\|^{2}+\frac{1}{\eta}\left\|\mathbf{b}^{n} \times \mathbf{u}^{n}\right\|^{2}+2 \eta\left\|\mathbf{b}^{n}\right\|^{2} .
\end{aligned}
$$

One estimates the momentum equation the same as for the Navier-Stokes equations, as in [11]. Fix any $\beta \in\left(\frac{1}{2}, 1\right)$ and let $C_{\beta}$ be $C$ as given by Theorem 2.1 with $\beta=\frac{1}{2}+\varepsilon$, so that we have the estimate

$$
\left\|\nabla p_{\mathrm{S}}\left(\mathbf{u}^{n}\right)\right\|^{2} \leq \beta\left\|\Delta \mathbf{u}^{n}\right\|^{2}+C_{\beta}\left\|\nabla \mathbf{u}^{n}\right\|^{2} .
$$

After testing (3.9) against $-\Delta \mathbf{u}^{n+1}$ and using (3.14) and that $\|\mathcal{P}\| \leq 1$, we estimate the right-hand side by

$$
\begin{aligned}
& \left|\left\langle\Delta \mathbf{u}^{n+1},-\nu p_{\mathrm{S}}\left(\mathbf{u}^{n}\right)+\mathcal{P} \mathbf{f}_{\text {tot }}^{n}\right\rangle\right| \\
\leq & \left\|\Delta \mathbf{u}^{n+1}\right\|\left(\left\|\nu p_{\mathrm{S}}\left(\mathbf{u}^{n}\right)\right\|+\left\|\mathbf{f}^{n}\right\|+\left\|\mathbf{u}^{n} \cdot \nabla \mathbf{u}^{n}\right\|+\left\|\alpha\left(\nabla \times \mathbf{b}^{n}\right) \times \mathbf{b}^{n}\right\|\right) \\
\leq & \left(\frac{\nu}{2}+\frac{\varepsilon_{1}}{2}\right)\left\|\Delta \mathbf{u}^{n+1}\right\|^{2}+\frac{\nu}{2}\left\|p_{\mathrm{S}}\left(\mathbf{u}^{n}\right)\right\|^{2} \\
& +\frac{3}{2 \varepsilon_{1}}\left(\left\|\mathbf{f}^{n}\right\|^{2}+\left\|\mathbf{u}^{n} \cdot \nabla \mathbf{u}^{n}\right\|^{2}+\left\|\alpha\left(\nabla \times \mathbf{b}^{n}\right) \times \mathbf{b}^{n}\right\|^{2}\right)
\end{aligned}
$$


for any $\varepsilon_{1}>0$. Then using (3.23), one finds easily that

$$
\begin{aligned}
& \frac{1}{\Delta t}\left(\left\|\nabla \mathbf{u}^{n+1}\right\|^{2}-\left\|\nabla \mathbf{u}^{n}\right\|^{2}\right)+\left(\nu-\varepsilon_{1}\right)\left(\left\|\Delta \mathbf{u}^{n+1}\right\|^{2}-\left\|\Delta \mathbf{u}^{n}\right\|^{2}\right)+\left(\nu-\varepsilon_{1}-\nu \beta\right)\left\|\Delta \mathbf{u}^{n}\right\|^{2} \\
\leq & \frac{3}{\varepsilon_{1}}\left(\left\|\mathbf{f}^{n}\right\|^{2}+\alpha^{2}\left\|\left(\nabla \times \mathbf{b}^{n}\right) \times \mathbf{b}^{n}\right\|^{2}+\left\|\mathbf{u}^{n} \cdot \nabla \mathbf{u}^{n}\right\|^{2}\right)+\nu C \beta\left\|\nabla \mathbf{u}^{n}\right\|^{2} .
\end{aligned}
$$

2. Now we turn to estimate nonlinear terms in (3.25) and (3.22), using the calculus inequalities in section 2.3 and the fact that $H^{1}(\Omega)$ embeds into $L^{4}$ and $L^{6}$ :

$$
\|(\nabla \times \mathbf{b}) \times \mathbf{b}\|^{2} \leq \begin{cases}\|\mathbf{b}\|_{L^{4}}^{2}\|\nabla \mathbf{b}\|_{L^{4}}^{2} \leq C\|\mathbf{b}\|\|\nabla \mathbf{b}\|^{2}\|\nabla \mathbf{b}\|_{H^{1}} & (N=2), \\ \|\mathbf{b}\|_{L^{6}}^{2}\|\nabla \mathbf{b}\|_{L^{3}}^{2} \leq C\|\nabla \mathbf{b}\|^{3}\|\nabla \mathbf{b}\|_{H^{1}} & (N=3) .\end{cases}
$$

By the elliptic regularity estimates $\|\mathbf{b}\|_{H^{1}} \leq C\|\mathbf{b}\|_{V}$ and $\|\nabla \mathbf{b}\|_{H^{1}} \leq\|\mathbf{b}\|_{H^{2}} \leq C\|\mathbf{b}\|_{W}$, we conclude that for any $\varepsilon_{2}>0$,

$$
\|(\nabla \times \mathbf{b}) \times \mathbf{b}\|^{2} \leq \begin{cases}C\|\mathbf{b}\|\|\mathbf{b}\|_{V}^{2}\|\mathbf{b}\|_{W} \leq \varepsilon_{2}\|\mathbf{b}\|_{W}^{2}+4 C \varepsilon_{2}^{-1}\|\mathbf{b}\|^{2}\|\mathbf{b}\|_{V}^{4} & (N=2), \\ C\|\mathbf{b}\|_{V}^{3}\|\mathbf{b}\|_{W} \leq \varepsilon_{2}\|\mathbf{b}\|_{W}^{2}+4 C \varepsilon_{2}^{-1}\|\mathbf{b}\|_{V}^{6} & (N=3) .\end{cases}
$$

Next, we find

$$
\begin{aligned}
\|\nabla \times(\mathbf{b} \times \mathbf{u})\|^{2} & \leq \begin{cases}\|\mathbf{u}\|_{L^{4}}^{2}\|\nabla \mathbf{b}\|_{L^{4}}^{2}+\|\mathbf{b}\|_{L^{4}}^{2}\|\nabla \mathbf{u}\|_{L^{4}}^{2} & (N=2), \\
\|\mathbf{u}\|_{L^{6}}^{2}\|\nabla \mathbf{b}\|_{L^{3}}^{2}+\|\mathbf{b}\|_{L^{6}}^{2}\|\nabla \mathbf{u}\|_{L^{3}}^{2} & (N=2 \text { or } 3),\end{cases} \\
& \leq \begin{cases}C\left(\|\mathbf{u}\|\|\nabla \mathbf{u}\|\|\mathbf{b}\|_{V}\|\mathbf{b}\|_{W}+\|\mathbf{b}\|\|\mathbf{b}\|_{V}\|\nabla \mathbf{u}\|\|\Delta \mathbf{u}\|\right) & (N=2), \\
C\left(\|\nabla \mathbf{u}\|^{2}\|\mathbf{b}\|_{V}\|\mathbf{b}\|_{W}+\|\mathbf{b}\|_{V}^{2}\|\nabla \mathbf{u}\|\|\Delta \mathbf{u}\|\right) & (N=3),\end{cases} \\
& \leq \varepsilon_{2}\left(\|\mathbf{b}\|_{W}^{2}+\|\Delta \mathbf{u}\|^{2}\right)+\frac{4 C}{\varepsilon_{2}}\left(\|\nabla \mathbf{u}\|^{4}\|\mathbf{b}\|_{V}^{2}+\|\mathbf{b}\| V^{2}\|\nabla \mathbf{u}\|^{4}\right)
\end{aligned}
$$

the last line is due to Young's inequality $a^{4} b^{2}+a^{2} b^{4} \leq a^{6}+b^{6}$. We also have

$$
\begin{aligned}
\|\mathbf{b} \times \mathbf{u}\|^{2} & \leq\|\mathbf{u}\|_{L^{4}}^{2}\|\mathbf{b}\|_{L^{4}}^{2} \leq\|\mathbf{u}\|\|\nabla \mathbf{u}\|\|\mathbf{b}\|\|\nabla \mathbf{b}\| \\
& \leq C\left(\|\nabla \mathbf{u}\|^{4}+\|\mathbf{b}\|_{V}^{4}\right) \leq C\left(\|\nabla \mathbf{u}\|^{2}+\|\nabla \mathbf{u}\|^{6}+\|\mathbf{b}\|_{V}^{2}+\|\mathbf{b}\|_{V}^{6}\right) .
\end{aligned}
$$

Finally, the velocity advection term is estimated similarly, as in [11], by

$$
\left\|\mathbf{u}^{n} \cdot \nabla \mathbf{u}^{n}\right\|^{2} \leq \varepsilon_{2}\left\|\Delta \mathbf{u}^{n}\right\|^{2}+4 C \varepsilon_{2}^{-1}\left\|\nabla \mathbf{u}^{n}\right\|^{6} \quad(N=2 \text { or } 3) .
$$

3. We plug these estimates into (3.25) and (3.22) and take $\varepsilon_{1}, \varepsilon_{2}>0$ to satisfy

$$
\hat{\nu}:=\nu-\varepsilon_{1}-\nu \beta-\frac{3 \varepsilon_{2}}{\varepsilon_{1}}-\frac{\varepsilon_{2}}{\eta}>0, \quad \hat{\eta}:=\eta-\frac{\varepsilon_{2}}{\eta}-\frac{3 \varepsilon_{2} \alpha^{2}}{\varepsilon_{1}}>0 .
$$

We obtain

$$
\begin{aligned}
& \frac{1}{\Delta t}\left(\left\|\mathbf{b}^{n+1}\right\|_{V}^{2}+\left\|\nabla \mathbf{u}^{n+1}\right\|^{2}-\left\|\mathbf{b}^{n}\right\|_{V}^{2}-\left\|\nabla \mathbf{u}^{n}\right\|^{2}\right)+\hat{\eta}\left\|\mathbf{b}^{n}\right\|_{W}^{2}+\hat{\nu}\left\|\Delta \mathbf{u}^{n}\right\|^{2} \\
& +\eta\left(\left\|\mathbf{b}^{n+1}\right\|_{W}^{2}-\left\|\mathbf{b}^{n}\right\|_{W}^{2}\right)+\left(\nu-\varepsilon_{1}\right)\left(\left\|\Delta \mathbf{u}^{n+1}\right\|^{2}-\left\|\Delta \mathbf{u}^{n}\right\|^{2}\right) \\
\leq & \frac{3}{\varepsilon_{1}}\left\|\mathbf{f}^{n}\right\|^{2}+\left(\nu C_{\beta}+\frac{C}{\eta}\right)\left\|\nabla \mathbf{u}^{n}\right\|^{2}+\left(2 \eta+\frac{C}{\eta}\right)\left\|\mathbf{b}^{n}\right\|_{V}^{2} \\
& +\left(\frac{4 C}{\eta \varepsilon_{2}}+\frac{12 C}{\varepsilon_{1} \varepsilon_{2}}+\frac{C}{\eta}\right)\left\|\nabla \mathbf{u}^{n}\right\|^{6}+\left(\frac{4 C}{\eta \varepsilon_{2}}+\alpha^{2} \frac{12 C}{\varepsilon_{1} \varepsilon_{2}}+\frac{C}{\eta}\right)\left\|\mathbf{b}^{n}\right\|_{V}^{6} .
\end{aligned}
$$


A simple discrete Gronwall-type argument concludes the proof. Put

$$
\begin{aligned}
& z_{n}=\left\|\mathbf{b}^{n}\right\|_{V}^{2}+\left\|\nabla \mathbf{u}^{n}\right\|^{2}+\eta \Delta t\left\|\mathbf{b}^{n}\right\|_{W}^{2}+\left(\nu-\varepsilon_{1}\right) \Delta t\left\|\Delta \mathbf{u}^{n}\right\|^{2}, \\
& w_{n}=\hat{\eta}\left\|\mathbf{b}^{n}\right\|_{W}^{2}+\hat{\nu}\left\|\Delta \mathbf{u}^{n}\right\|^{2}, \quad b_{n}=\left\|\mathbf{f}^{n}\right\|^{2},
\end{aligned}
$$

and note that from (3.8) we have that as long as $n \Delta t \leq T$,

$$
\sum_{k=0}^{n-1}\left\|\mathbf{f}^{k}\right\|^{2} \Delta t \leq \int_{0}^{T}|\mathbf{f}(t)|^{2} d t
$$

by the Cauchy-Schwarz inequality. Then by (3.30), with $\varphi(z)=C\left(z+z^{3}\right)$, as long as $k \Delta t \leq T$ we have

$$
z_{k+1}+w_{k} \Delta t \leq z_{k}+\left(C b_{k}+\varphi\left(z_{k}\right)\right) \Delta t
$$

for a constant $C$ now depending on $\eta$ and $\nu$. Summing (3.34) from 0 to $n-1$ and using (3.33) yields

$$
z_{n}+\sum_{k=0}^{n-1} w_{k} \Delta t \leq y_{n}:=M+\sum_{k=0}^{n-1} \varphi\left(z_{k}\right) \Delta t
$$

where $M=C M_{0}$. Next we apply the following general lemma (which determines $T_{*}$ ). LEMmA 3.2. Let $\varphi:(0, \infty) \rightarrow(0, \infty)$ be continuous and increasing, and let $M>0$. Given any $T_{*}$ such that $0<T_{*}<\int_{M}^{\infty} d z / \varphi(z)$, there exists $C_{*}>0$ independent of $\Delta t>0$ with the following property. Suppose that quantities $z_{n}, w_{n} \geq 0$ satisfy (3.35) for $n=0,1, \ldots, n_{*}$, with $n_{*} \Delta t \leq T_{*}$. Then $y_{n_{*}} \leq C_{*}$ (independent of $\left.\Delta t\right)$.

Proof. The quantities $y_{n}$ defined in (3.35) increase with $n$ and satisfy

$$
y_{n+1}-y_{n}=\varphi\left(z_{n}\right) \Delta t \leq \varphi\left(y_{n}\right) \Delta t .
$$

Let $F(y)=\int_{M}^{y} d z / \varphi(z)$. Then $F^{\prime}(y)=1 / \varphi(y)$ is positive and decreasing, and we have

$$
F\left(y_{n+1}\right)-F\left(y_{n}\right) \leq F^{\prime}\left(y_{n}\right)\left(y_{n+1}-y_{n}\right)=\frac{y_{n+1}-y_{n}}{\varphi\left(y_{n}\right)} \leq \Delta t
$$

whence $F\left(y_{n}\right) \leq F\left(y_{0}\right)+n \Delta t=n \Delta t$, since $y_{0}=M$. Now, $0<T_{*}<F(\infty)=\sup _{y>0} F(y)$ (which may be finite or infinite), so as long as $n \Delta t \leq T_{*}$ we have $y_{n} \leq C_{*}=F^{-1}\left(T_{*}\right)$. This finishes the proof of the lemma.

By (3.35) this result yields the stability estimate (3.15). Now, using (3.29) and elliptic regularity, we obtain from (3.15) that

$$
\begin{aligned}
\sum_{k=0}^{n}\left\|\nabla \times\left(\mathbf{b}^{k} \times \mathbf{u}^{k}\right)\right\|^{2} \Delta t & \leq C \sum_{k=0}^{n}\left(\left\|\mathbf{b}^{k}\right\|_{V}^{2}\left\|\Delta \mathbf{u}^{k}\right\|^{2}+\left\|\nabla \mathbf{u}^{k}\right\|^{2}\left\|\mathbf{b}^{k}\right\|_{W}^{2}\right) \Delta t \\
& \leq C \sum_{k=0}^{n}\left(\left\|\Delta \mathbf{u}^{k}\right\|^{2}+\left\|\mathbf{b}^{k}\right\|_{W}^{2}\right) \Delta t \leq C .
\end{aligned}
$$

Similarly, one finds

$$
\left.\sum_{k=0}^{n}\left(\|\left(\nabla \times \mathbf{b}^{k}\right) \times \mathbf{b}^{k}\right)\left\|^{2}+\right\| \mathbf{u}^{k} \cdot \nabla \mathbf{u}^{k} \|^{2}\right) \Delta t \leq C,
$$


giving (3.16). Then the difference equations (3.9)-(3.10) yield

$$
\sum_{k=0}^{n-1}\left(\left\|\frac{\mathbf{b}^{k+1}-\mathbf{b}^{k}}{\Delta t}\right\|^{2}+\left\|\frac{\mathbf{u}^{k+1}-\mathbf{u}^{k}}{\Delta t}\right\|^{2}\right) \Delta t \leq C .
$$

This yields (3.17) and finishes the proof of the Theorem.

REMARK 3.1. The value of $T_{*}$ in the proof is limited by combined effects of nonlinearity, viscosity, and magnetic resistivity. If the nonlinear terms in the MHD system are replaced by linearization about suitably regular velocity and magnetic fields, then the bound in (3.34) is obtained with $\varphi(z)=C z$. Then one can take $T_{*}$ arbitrarily large, and this indicates unconditional stability for the time-discrete scheme.

3.4. Approximation of initial data. According to our hypotheses, we take the initial data in (1.9) to satisfy $\mathbf{u}_{\text {in }}, \mathbf{b}_{\text {in }} \in H^{1}\left(\Omega, \mathbb{R}^{N}\right)$ with

$$
\mathbf{n} \cdot \mathbf{u}_{\text {in }}=0, \quad \mathbf{n} \cdot \mathbf{b}_{\text {in }}=0 \quad \text { on } \Gamma .
$$

Given $\Delta t>0$, it is convenient to determine $\mathbf{u}^{0}$ in $H_{0}^{1} \cap H^{2}\left(\Omega, \mathbb{R}^{N}\right)$ by solving ( $I-$ $\Delta t \Delta) \mathbf{u}^{0}=\mathbf{u}_{\text {in }}$. An energy estimate yields

$$
\left\|\nabla \mathbf{u}^{0}\right\|^{2}+\Delta t\left\|\Delta \mathbf{u}^{0}\right\|^{2}=\left\langle\nabla \mathbf{u}_{\text {in }}, \nabla \mathbf{u}^{0}\right\rangle \leq\left\|\nabla \mathbf{u}_{\text {in }}\right\|\left\|\nabla \mathbf{u}^{0}\right\| \leq\left\|\nabla \mathbf{u}_{\text {in }}\right\|^{2} .
$$

Then $\left\|\Delta t \Delta \mathbf{u}^{0}\right\|^{2}=O(\Delta t)$ as $\Delta t \rightarrow 0$, so $\mathbf{u}^{0} \rightarrow \mathbf{u}_{\text {in }}$ strongly in $L^{2}$ and weakly in $H^{1}$.

In a similar way, using Lemma 2.2 we determine $\mathbf{b}^{0}$ in $H^{2}\left(\Omega, \mathbb{R}^{N}\right)$ by solving

$$
(I-\Delta t \Delta) \mathbf{b}^{0}=\mathbf{b}_{\text {in }},\left.\quad \mathbf{n} \cdot \mathbf{b}^{0}\right|_{\Gamma}=0, \quad \mathbf{n} \times\left.\left(\nabla \times \mathbf{b}^{0}\right)\right|_{\Gamma}=0 .
$$

Then $\mathbf{b}^{0} \in W$. An energy estimate yields

$$
\begin{aligned}
\left\|\nabla \cdot \mathbf{b}^{0}\right\|^{2}+\left\|\nabla \times \mathbf{b}^{0}\right\|^{2}+\Delta t\left\|\Delta \mathbf{b}^{0}\right\|^{2} & =-\left\langle\Delta \mathbf{b}^{0}, \mathbf{b}_{\text {in }}\right\rangle \\
& =\left\langle\nabla \cdot \mathbf{b}^{0}, \nabla \cdot \mathbf{b}_{\text {in }}\right\rangle+\left\langle\nabla \times \mathbf{b}^{0}, \nabla \times \mathbf{b}_{\text {in }}\right\rangle .
\end{aligned}
$$

Hence

$$
\left\|\nabla \cdot \mathbf{b}^{0}\right\|^{2}+\left\|\nabla \times \mathbf{b}^{0}\right\|^{2}+2 \Delta t\left\|\Delta \mathbf{b}^{0}\right\|^{2} \leq\left\|\nabla \cdot \mathbf{b}_{\text {in }}\right\|^{2}+\left\|\nabla \times \mathbf{b}_{\text {in }}\right\|^{2} .
$$

Then $\left\|\Delta t \Delta \mathbf{b}^{0}\right\|^{2}=O(\Delta t)$ as $\Delta t \rightarrow 0$, so $\mathbf{b}^{0} \rightarrow \mathbf{b}_{\text {in }}$ strongly in $L^{2}$ and weakly in $H^{1}$.

For later use, we remark that multiplying the first equation in (3.40) by $-\nabla \nabla \cdot \mathbf{b}^{0}$ and integration by parts gives an estimate on the divergence of $\mathbf{b}^{0}$ :

$$
\left\|\nabla \cdot \mathbf{b}^{0}\right\|^{2}+\Delta t\left\|\nabla \nabla \cdot \mathbf{b}^{0}\right\|^{2}=\left\langle\nabla \cdot \mathbf{b}^{0}, \nabla \cdot \mathbf{b}_{\text {in }}\right\rangle \leq\left\|\nabla \cdot \mathbf{b}^{0}\right\|\left\|\nabla \cdot \mathbf{b}_{\text {in }}\right\| \leq\left\|\nabla \cdot \mathbf{b}_{\text {in }}\right\|^{2} .
$$

3.5. Homogenizing the boundary conditions. We proceed next to consider the case of general boundary data $\mathbf{g}$ having the regularity indicated in (1.14), and initial data for velocity with regularity $\mathbf{u}_{\text {in }} \in H_{\text {in }}:=H^{1}\left(\Omega, \mathbb{R}^{N}\right)$. We also assume the compatibility conditions (1.15) hold. The space in which we seek strong solutions is

$$
V(0, T):=L^{2}\left(0, T ; H^{2}(\Omega)\right) \cap H^{1}\left(0, T ; L^{2}(\Omega)\right) .
$$

From the theory of Lions and Magenes [14] (see Theorems 2.3 and 4.3 in vol. II), taking the trace on the parabolic boundary of $\Omega \times(0, T)$, defined for smooth enough functions by $u \mapsto\left(u(\cdot, 0),\left.u\right|_{\Gamma}\right)$, extends to yield a bounded map

$$
\begin{gathered}
V(0, T) \rightarrow H^{1}(\Omega) \times\left(H^{3 / 4}\left(0, T ; L^{2}(\Gamma)\right) \cap L^{2}\left(0, T ; H^{3 / 2}(\Gamma)\right)\right) \\
\cap\{(u, g) \mid u=g \text { on } \Gamma \text { for } t=0\},
\end{gathered}
$$


and this map admits a bounded right inverse. By consequence, given $\left(\mathbf{u}_{\mathrm{in}}, \mathbf{g}\right)$ satisfying our assumptions above, there exists $\tilde{\mathbf{u}}$ such that

$$
\tilde{\mathbf{u}} \in V(0, T)^{N}, \quad \tilde{\mathbf{u}}(0)=\mathbf{u}_{\text {in }},\left.\quad \tilde{\mathbf{u}}\right|_{\Gamma}=\mathbf{g}
$$

and the norm of $\tilde{\mathbf{u}}$ in $V(0, T)^{N}$ is bounded in terms of the norm of $\left(\mathbf{u}_{\text {in }}, \mathbf{g}\right)$ in $H_{\text {in }} \times H_{g}$. One can regard $\tilde{\mathbf{u}}$ as given data, instead of the pair $\left(\mathbf{u}_{\mathrm{in}}, \mathbf{g}\right)$.

We define $\mathbf{v}=\mathbf{u}-\tilde{\mathbf{u}}$. Then $\mathbf{v}(\cdot, 0)=0$ in $\Omega$ and $\mathbf{v}=0$ on $\Gamma$. We can rewrite $(2.10)$ as an equation for $\mathbf{v}$ :

$$
\frac{\partial \mathbf{v}}{\partial t}+\nu \nabla p_{\mathrm{S}}(\mathbf{v})=\nu \Delta \mathbf{v}+\mathcal{P} \hat{\mathbf{f}}_{\mathrm{tot}}-\mathcal{P}(\mathbf{v} \cdot \nabla \tilde{\mathbf{u}}+\tilde{\mathbf{u}} \cdot \nabla \mathbf{v})-\tilde{\mathbf{f}},
$$

where

$$
\begin{aligned}
& \hat{\mathbf{f}}_{\mathrm{tot}}=-\mathbf{v} \cdot \nabla \mathbf{v}+\alpha(\nabla \times \mathbf{b}) \times \mathbf{b}+\mathbf{f}, \\
& \tilde{\mathbf{f}}=\partial_{t} \tilde{\mathbf{u}}+\nu \nabla p_{\mathrm{S}}(\tilde{\mathbf{u}})-\nu \Delta \tilde{\mathbf{u}}+\mathcal{P}(\tilde{\mathbf{u}} \cdot \nabla \tilde{\mathbf{u}}) .
\end{aligned}
$$

3.6. Stability analysis for non-homogeneous boundary conditions. We assume the data satisfy (1.12)-(1.14) for some given $T>0$, together with (1.15). To prove the stability and convergence of the discretization scheme, we use $\tilde{\mathbf{u}}$ which satisfies (3.44) and is bounded in terms of $\left(\mathbf{u}_{\text {in }}, \mathbf{g}\right)$. We define

$$
\tilde{\mathbf{u}}^{n}=\frac{1}{\Delta t} \int_{n \Delta t}^{(n+1) \Delta t} \tilde{\mathbf{u}}(t) d t, \quad \mathbf{v}^{n}=\mathbf{u}^{n}-\tilde{\mathbf{u}}^{n},
$$

and we assume that $\mathbf{u}^{0}=\tilde{\mathbf{u}}^{0}$. Then $\mathbf{v}^{0}=0$ in $\Omega$, and $\mathbf{v}^{n}=0$ on $\Gamma$ for $n \geq 0$. We can rewrite (3.9) as an equation for $\mathbf{v}^{n}$ :

$$
\frac{\mathbf{v}^{n+1}-\mathbf{v}^{n}}{\Delta t}-\nu \Delta \mathbf{v}^{n+1}=-\nu \nabla p_{\mathrm{S}}\left(\mathbf{v}^{n}\right)+\mathcal{P} \hat{\mathbf{f}}_{\text {tot }}^{n}-\mathcal{P}\left(\mathbf{v}^{n} \cdot \nabla \tilde{\mathbf{u}}^{n}+\tilde{\mathbf{u}}^{n} \cdot \nabla \mathbf{v}^{n}\right)-\tilde{\mathbf{f}}^{n},
$$

where

$$
\begin{aligned}
& \hat{\mathbf{f}}_{\mathrm{tot}}^{n}=-\mathbf{v}^{n} \cdot \nabla \mathbf{v}^{n}+\alpha\left(\nabla \times \mathbf{b}^{n}\right) \times \mathbf{b}^{n}+\mathbf{f}^{n}, \\
& \tilde{\mathbf{f}}^{n}=\frac{\tilde{\mathbf{u}}^{n+1}-\tilde{\mathbf{u}}^{n}}{\Delta t}+\nu \nabla p_{\mathrm{S}}\left(\tilde{\mathbf{u}}^{n}\right)-\nu \Delta \tilde{\mathbf{u}}^{n+1}+\mathcal{P}\left(\tilde{\mathbf{u}}^{n} \cdot \nabla \tilde{\mathbf{u}}^{n}\right) .
\end{aligned}
$$

Equation (3.10) and the boundary conditions for $\mathbf{b}^{n}$ in (3.11) remain unchanged.

We claim there exists $M_{*}>0$ depending only on the norm of the data $\left(\mathbf{u}_{\text {in }}, \mathbf{g}\right)$ such that, for $(n+1) \Delta t \leq T$,

$$
\sum_{k=0}^{n-1}\left\|\tilde{\mathbf{f}}^{k}\right\|^{2} \Delta t \leq M_{*}
$$

Because of the embedding $\tilde{\mathbf{u}} \in V(0, T)^{N} \hookrightarrow C\left([0, T], H^{1}\left(\Omega, \mathbb{R}^{N}\right)\right)$ (see $[16$, p. 42$]$ or $[5$, p. 288]) and

$$
\frac{\tilde{\mathbf{u}}^{n+1}-\tilde{\mathbf{u}}^{n}}{\Delta t}=\int_{t_{n}}^{t_{n+1}} \int_{0}^{\Delta t} \partial_{t} \tilde{\mathbf{u}}(t+s) \frac{d s}{\Delta t} \frac{d t}{\Delta t}=\int_{0}^{2} \partial_{t} \tilde{\mathbf{u}}\left(t_{n}+\tau \Delta t\right) \Lambda(\tau) d \tau
$$


where $\Lambda(\tau)=1-|1-\tau|$, due to the Cauchy-Schwarz inequality we have

$$
\begin{array}{r}
\sup _{0 \leq k \leq n}\left\|\tilde{\mathbf{u}}^{k}\right\|_{H^{1}}^{2}+\sum_{k=0}^{n}\left\|\tilde{\mathbf{u}}^{k}\right\|_{H^{2}}^{2} \Delta t+\sum_{k=1}^{n-1}\left\|\frac{\tilde{\mathbf{u}}^{n+1}-\tilde{\mathbf{u}}^{n}}{\Delta t}\right\|^{2} \Delta t \\
\leq C\|\tilde{\mathbf{u}}\|_{V(0, T)}^{2} \leq C\left(\left\|\mathbf{u}_{\text {in }}\right\|_{H^{1}}^{2}+\|\mathbf{g}\|_{H_{g}}^{2}\right) .
\end{array}
$$

Using this with (3.29) bounds the nonlinear term in (3.47). Thus we obtain the bound (3.52).

Following the approach of subsection 3.3, we obtain an extension of Theorem 3.1.

THEOREM 3.3. Let $\Omega$ be a bounded domain in $\mathbb{R}^{N}$ ( $N=2$ or 3 ) with smooth boundary, and let $\nu, \eta, M_{0}>0$. Then there exist positive constants $T_{*}$ and $C_{3}$, such that if (1.12)-(1.15) hold for some $T>0$, with

$$
\left\|\mathbf{u}_{\text {in }}\right\|_{H^{1}}^{2}+\left\|\mathbf{b}_{\text {in }}\right\|_{H^{1}}^{2}+\int_{0}^{T}\|\mathbf{f}(t)\|^{2} d t+\|\mathbf{g}\|_{H_{g}}^{2} \leq M_{0},
$$

then whenever $2 \eta \Delta t \leq 1$ and $(n+1) \Delta t \leq T \leq T_{*}$, the solution to the time-discrete scheme (3.9)-(3.13), with $\mathbf{u}^{0}=\tilde{\mathbf{u}}^{0}$ from (3.48) and (3.44), and $\mathbf{b}^{0}$ given by (3.40), satisfies

$$
\begin{aligned}
& \sup _{0 \leq k \leq n}\left(\left\|\mathbf{b}^{k}\right\|_{H^{1}}^{2}+\left\|\mathbf{u}^{k}\right\|_{H^{1}}^{2}\right)+\sum_{k=0}^{n}\left(\left\|\mathbf{b}^{k}\right\|_{H^{2}}^{2}+\left\|\mathbf{u}^{k}\right\|_{H^{2}}^{2}\right) \Delta t \leq C_{3}, \\
& \left.\sum_{k=0}^{n-1}\left(\left\|\nabla \times\left(\mathbf{b}^{k} \times \mathbf{u}^{k}\right)\right\|^{2}+\|\left(\nabla \times \mathbf{b}^{k}\right) \times \mathbf{b}^{k}\right)\left\|^{2}+\right\| \mathbf{u}^{k} \cdot \nabla \mathbf{u}^{k} \|^{2}\right) \Delta t \leq C_{3} . \\
& \sum_{k=0}^{n-1}\left(\left\|\frac{\mathbf{b}^{k+1}-\mathbf{b}^{k}}{\Delta t}\right\|^{2}+\left\|\frac{\mathbf{u}^{k+1}-\mathbf{u}^{k}}{\Delta t}\right\|^{2}\right) \Delta t \leq C_{3} .
\end{aligned}
$$

Inequalities (3.55)-(3.57) are also true with $\mathbf{u}^{k}$ replaced by $\mathbf{v}^{k}$ as given by (3.48).

Proof. We first write (3.9) as (3.49). Using (3.52) and comparing with the proof of Theorem 3.1, we see that the only essential difference is that in (3.49) we have some extra linear terms of the form

$$
\mathcal{P}\left(\tilde{\mathbf{u}}^{n} \cdot \nabla \mathbf{v}^{n}+\mathbf{v}^{n} \cdot \nabla \tilde{\mathbf{u}}^{n}\right),
$$

and the term $\tilde{\mathbf{f}}^{n}$. Similar to (3.29), we obtain

$$
\|\mathcal{P}(\tilde{\mathbf{u}} \cdot \nabla \mathbf{v})\|^{2} \leq \varepsilon\|\Delta \mathbf{v}\|^{2}+\frac{C}{\varepsilon}\|\tilde{\mathbf{u}}\|_{H^{1}}^{4}\|\nabla \mathbf{v}\|^{2} .
$$

We estimate the other term in (3.58) by using Gagliardo-Nirenberg inequalities [6, Thm. 10.1] and the Sobolev embeddings of $H^{1}$ into $L^{3}$ and $L^{6}$ :

$$
\|\mathbf{v}\|_{L^{\infty}} \leq \begin{cases}C\|\Delta \mathbf{v}\|_{L^{3 / 2}}^{1 / 2}\|\mathbf{v}\|_{L^{3}}^{1 / 2} \leq C\|\Delta \mathbf{v}\|^{1 / 2}\|\nabla \mathbf{v}\|^{1 / 2} & (N=2), \\ C\|\Delta \mathbf{v}\|^{1 / 2}\|\mathbf{v}\|_{L^{6}}^{1 / 2} \leq C\|\Delta \mathbf{v}\|^{1 / 2}\|\nabla \mathbf{v}\|^{1 / 2} & (N=3) .\end{cases}
$$

Then for $N=2$ and 3 we have

$$
\|\mathcal{P}(\mathbf{v} \cdot \nabla \tilde{\mathbf{u}})\|^{2} \leq\|\mathbf{v}\|_{L^{\infty}}^{2}\|\nabla \tilde{\mathbf{u}}\|^{2} \leq \varepsilon\|\Delta \mathbf{v}\|^{2}+\frac{C}{\varepsilon}\|\tilde{\mathbf{u}}\|_{H^{1}}^{4}\|\nabla \mathbf{v}\|^{2} .
$$

With these estimates, the rest of the proof of the stability of $\mathbf{v}^{n}$ is essentially the same as that of Theorem 3.1 and therefore we omit the details. The stability of $\mathbf{v}^{n}$ leads to that of $\mathbf{u}^{n}$, using (3.54). 


\section{Existence, uniqueness, convergence}

For the constrained MHD equations that include the divergence-free conditions (1.3)-(1.4), in which pressure is determined accordingly, local existence and uniqueness of strong solutions with no-slip boundary condition is classical, see [4]. Here we will extend the local existence and uniqueness theory to treat the unconstrained formulation of the MHD equations (1.1), (1.2) with pressure given by (2.11), intial conditions (1.9), and boundary conditions (1.5) and (1.8). The stability estimates in Theorem 3.3 lead to a standard compactness proof for existence of a strong solution. The estimates in the stability argument, based on Theorem 2.1 in particular, also permit a simple uniqueness proof. Full convergence of the time-discrete scheme (3.9)-(3.13) follows as a consequence.

THEOREM 4.1. Let $\Omega$ be a bounded domain in $\mathbb{R}^{N}(N=2$ or 3$)$ with a smooth boundary $\Gamma$, and let $\nu, \eta, M_{1}>0$. Then, there exists $T_{*}>0$ such that if the data satisfy (1.12)-(1.15) for any $T \in\left(0, T_{*}\right]$, with

$$
\left\|\mathbf{u}_{\text {in }}\right\|_{H^{1}}+\left\|\mathbf{b}_{\text {in }}\right\|_{H^{1}}+\|\mathbf{f}\|_{L^{2}\left(0, T, L^{2}\left(\Omega, \mathbb{R}^{N}\right)\right)}+\|\mathbf{g}\|_{H_{g}} \leq M_{1},
$$

then a unique strong solution of (1.1), (1.2), and (2.11) exists on $[0, T]$ that satisfies the conditions (1.5), (1.8), and (1.9) and has the regularity indicated in (1.10)-(1.11), and thus $\mathbf{u}, \mathbf{b} \in C\left([0, T], H^{1}\left(\Omega, \mathbb{R}^{N}\right)\right)$.

Moreover, for $t>0, \nabla \cdot \mathbf{u}$ and $\nabla \cdot \mathbf{b}$ are $C^{\infty}$ classical solutions of the diffusion equations with no-flux boundary conditions (3.3) and (3.7), respectively. The maps $t \mapsto\|\nabla \cdot \mathbf{u}\|^{2}$ and $t \mapsto\|\nabla \cdot \mathbf{b}\|^{2}$ are smooth for $t>0$ and we have the dissipation identities

$$
\begin{aligned}
& \frac{d}{d t} \frac{1}{2}\|\nabla \cdot \mathbf{u}\|^{2}+\nu\|\nabla(\nabla \cdot \mathbf{u})\|^{2}=0, \\
& \frac{d}{d t} \frac{1}{2}\|\nabla \cdot \mathbf{b}\|^{2}+\eta\|\nabla(\nabla \cdot \mathbf{b})\|^{2}=0 .
\end{aligned}
$$

We will not give the full existence proof, since the compactness method is classical $[16,17,14]$ and the details are similar to the treatment of an unconstrained formulation of the Navier-Stokes equations in [11]. The main steps are: (i) piecewise linear interpolation of the time-discrete scheme, (ii) using the stability estimates of Theorem 3.3 to extract weakly convergent subsequences, (iii) using strong convergence in $L^{2}([0, T] \times \Omega)$ to establish convergence of nonlinear terms in the sense of distributions. Passing to the limit, one shows the velocity is a strong solution of

$$
\partial_{t} \mathbf{u}=\nu \nabla \nabla \cdot \mathbf{u}+\mathcal{P}\left(\nu \Delta \mathbf{u}+\mathbf{f}_{\mathrm{tot}}\right)
$$

which is equivalent to (2.10) by (2.6) and (2.4). Here we will not discuss the details, and refer to [11]. We proceed to address the uniqueness and the properties of $\nabla \cdot \mathbf{u}$ and $\nabla \cdot \mathbf{b}$.

\subsection{Uniqueness for unconstrained MHD equations.}

Proof of uniqueness: Recall the definition of $V(0, T)$ from (3.42). Suppose $\mathbf{u}_{1}, \mathbf{b}_{1} \in$ $V(0, T)^{N}$ and $\mathbf{u}_{2}, \mathbf{b}_{2} \in V(0, T)^{N}$ are both solutions of (1.1), (1.2), and (2.12), satisfying the boundary conditions (1.5), (1.8) and the initial conditions (1.9).

Put $\mathbf{u}=\mathbf{u}_{1}-\mathbf{u}_{2}, \mathbf{b}=\mathbf{b}_{1}-\mathbf{b}_{2}$ and $p=p_{\mathrm{S}}(\mathbf{u})$. Then $\mathbf{u}(0)=\mathbf{b}(0)=0$ and

$$
\partial_{t} \mathbf{u}+\mathcal{P}\left(\mathbf{u}_{1} \cdot \nabla \mathbf{u}+\mathbf{u} \cdot \nabla \mathbf{u}_{2}\right)=\nu \Delta \mathbf{u}-\nu \nabla p+\alpha \mathcal{P}\left(\left(\nabla \times \mathbf{b}_{1}\right) \times \mathbf{b}+(\nabla \times \mathbf{b}) \times \mathbf{b}_{2}\right),
$$




$$
\partial_{t} \mathbf{b}+\nabla \times\left(\mathbf{b}_{1} \times \mathbf{u}+\mathbf{b} \times \mathbf{u}_{2}\right)=\eta \Delta \mathbf{b}
$$

with boundary conditions

$$
\mathbf{u}=0, \quad \mathbf{n} \cdot \mathbf{b}=0, \quad \mathbf{n} \times(\nabla \times \mathbf{b})=0 .
$$

Dot (4.4) with $-\Delta \mathbf{u}$ and $\operatorname{dot}(4.5)$ with $\mathbf{b}-\Delta \mathbf{b}$. Due to the boundary conditions (4.6), we infer that the quantities $\left\langle\partial_{t} \mathbf{u},-\Delta \mathbf{u}\right\rangle$ and $\left\langle\partial_{t} \mathbf{b}, \mathbf{b}-\Delta \mathbf{b}\right\rangle$ are in $L^{1}(0, T)$ and $t \mapsto\|\nabla \mathbf{u}\|^{2}+\|\mathbf{b}\|_{V}^{2}$ is absolutely continuous with

$$
\left\langle\partial_{t} \mathbf{u},-\Delta \mathbf{u}\right\rangle+\left\langle\partial_{t} \mathbf{b}, \mathbf{b}-\Delta \mathbf{b}\right\rangle=\frac{1}{2} \frac{d}{d t}\left(\|\nabla \mathbf{u}\|^{2}+\|\mathbf{b}\|_{V}^{2}\right) .
$$

This can be justified using approximation by smooth functions; Evans [5, p. 287] provides a detailed proof of a similar result.

We estimate remaining terms as follows. Using Theorem 2.1 we obtain

$$
\langle\nu \Delta \mathbf{u}-\nu \nabla p,-\Delta \mathbf{u}\rangle \leq-\frac{\nu}{2}\|\Delta \mathbf{u}\|^{2}+\frac{\nu}{2}\|\nabla p\|^{2} \leq-\frac{\nu \beta}{2}\|\Delta \mathbf{u}\|^{2}+C\|\nabla \mathbf{u}\|^{2} .
$$

with $\beta=\frac{1}{2}-\varepsilon>0$. Next, use the Cauchy-Schwarz inequality for the nonlinear terms, estimating them as follows in a manner similar to step 2 in the proof of Theorem 3.1, using that $\mathbf{u}_{1}, \mathbf{u}_{2}, \mathbf{b}_{1}, \mathbf{b}_{2}$ are a priori bounded in $H^{1}$ norm:

$$
\begin{gathered}
\left\|\mathbf{u}_{1} \cdot \nabla \mathbf{u}\right\|\|\Delta \mathbf{u}\| \leq C\left\|\nabla \mathbf{u}_{1}\right\|\|\nabla \mathbf{u}\|^{1 / 2}\|\Delta \mathbf{u}\|^{3 / 2} \leq \varepsilon\|\Delta \mathbf{u}\|^{2}+C\|\nabla \mathbf{u}\|^{2} \\
\left\|\mathbf{u} \cdot \nabla \mathbf{u}_{2}\right\|\|\Delta \mathbf{u}\| \leq C\|\nabla \mathbf{u}\|\left\|\nabla \mathbf{u}_{2}\right\|_{H^{1}}\|\Delta \mathbf{u}\| \leq \varepsilon\|\Delta \mathbf{u}\|^{2}+C\left\|\mathbf{u}_{2}\right\|_{H^{2}}^{2}\|\nabla \mathbf{u}\|^{2} \\
\left\|(\nabla \times \mathbf{b}) \times \mathbf{b}_{2}\right\|\|\Delta \mathbf{u}\| \leq C\left\|\mathbf{b}_{2}\right\|_{H^{1}}\|\nabla \mathbf{b}\|^{1 / 2}\|\mathbf{b}\|_{H^{2}}^{1 / 2}\|\Delta \mathbf{u}\| \\
\quad \leq \varepsilon\left(\|\Delta \mathbf{u}\|^{2}+\|\mathbf{b}\|_{H^{2}}^{2}\right)+C\|\mathbf{b}\|_{H^{1}}^{2} \\
\left\|\left(\nabla \times \mathbf{b}_{1}\right) \times \mathbf{b}\right\|\|\Delta \mathbf{u}\| \leq C\|\mathbf{b}\|_{H^{1}}\left\|\nabla \mathbf{b}_{1}\right\|_{H^{1}}\|\Delta \mathbf{u}\| \leq \varepsilon\|\Delta \mathbf{u}\|^{2}+C\left\|_{2}\right\|_{H^{2}}^{2}\|\mathbf{b}\|_{H^{1}}^{2}, \\
\left\|\nabla \times\left(\mathbf{b}_{1} \times \mathbf{u}\right)\right\|\|\mathbf{b}-\Delta \mathbf{b}\| \leq \varepsilon\left(\|\Delta \mathbf{u}\|^{2}+\|\mathbf{b}\|_{H^{2}}^{2}\right)+C\|\nabla \mathbf{u}\|^{2}\left(1+\left\|\mathbf{b}_{1}\right\|_{H^{2}}^{2}\right) \\
\left\|\nabla \times\left(\mathbf{b} \times \mathbf{u}_{2}\right)\right\|\|\mathbf{b}-\Delta \mathbf{b}\| \leq \varepsilon\|\mathbf{b}\|_{H^{2}}^{2}+C\left(1+\left\|\Delta \mathbf{u}_{2}\right\|^{2}\right)\|\nabla \mathbf{b}\|^{2}
\end{gathered}
$$

This gives

$$
\begin{aligned}
& \frac{d}{d t}\left(\|\nabla \mathbf{u}\|^{2}+\|\mathbf{b}\|_{V}^{2}\right)+\nu\|\Delta \mathbf{u}\|^{2}+\eta\|\mathbf{b}\|_{W}^{2} \\
\leq & C\left(1+\left\|\mathbf{b}_{1}\right\|_{H^{2}}^{2}+\left\|\mathbf{b}_{2}\right\|_{H^{2}}^{2}+\left\|\Delta \mathbf{u}_{2}\right\|^{2}\right)\left(\|\nabla \mathbf{u}\|^{2}+\|\mathbf{b}\|_{V}^{2}\right) .
\end{aligned}
$$

Because $\left\|\mathbf{b}_{1}\right\|_{H^{2}}^{2},\left\|\mathbf{b}_{2}\right\|_{H^{2}}^{2}$, and $\left\|\Delta \mathbf{u}_{2}\right\|^{2}$ are in $L^{1}(0, T)$, by Gronwall's inequality we obtain $\|\nabla \mathbf{u}\|^{2}+\|\mathbf{b}\|_{V}^{2} \equiv 0$. This finishes the proof of uniqueness.

4.2. Divergence of the velocity and magnetic fields. It remains to discuss the properties stated in Theorem 4.1 regarding the regularity and behavior of $\nabla \cdot \mathbf{u}$ and $\nabla \cdot \mathbf{b}$ for $t>0$. The first step is to observe that since $\mathbf{u}$ and $\mathbf{b}$ are strong solutions on $(0, T)$, for any $\phi \in H^{1}(\Omega)$ the computations leading to the weak-form Equ. (3.2) and (3.6) are valid in $L^{1}(0, T)$. Then just as in the proof for the Navier-Stokes equations in [11], the smoothness of $\nabla \cdot \mathbf{u}$ and $\nabla \cdot \mathbf{b}$ follow from semigroup theory, using Ball's characterization of weak solutions of abstract evolution equations [2]. The dissipation identities (4.1), (4.2) follow by dotting (3.3) and (3.7) with $\nabla \cdot \mathbf{u}$ and $\nabla \cdot \mathbf{b}$ respectively. 


\section{Time-discrete divergence estimate}

In this section we estimate the divergence of the time-discrete velocity and magnetic field for the scheme (3.9)-(3.13). The main results are the bounds in (5.2) and (5.10) below.

Consider the magnetic field first. Take a test function $\phi \in H^{1}(\Omega)$ with mean zero, and $\operatorname{dot}(3.10)$ with $\nabla \phi$. As in section 3.1, we find

$$
\left\langle\frac{\mathbf{b}^{n+1}-\mathbf{b}^{n}}{\Delta t}, \nabla \phi\right\rangle=\eta\left\langle\nabla\left(\nabla \cdot \mathbf{b}^{n+1}\right), \nabla \phi\right\rangle
$$

Taking $\phi=\nabla \cdot \mathbf{b}^{n+1}$, integrating the time difference term by parts, and summing, one infers

$$
\left\|\nabla \cdot \mathbf{b}^{n}\right\|^{2}+\nu \sum_{k=1}^{n}\left\|\nabla \nabla \cdot \mathbf{b}^{k}\right\|^{2} \Delta t \leq\left\|\nabla \cdot \mathbf{b}^{0}\right\|^{2} \leq\left\|\nabla \cdot \mathbf{b}_{\text {in }}\right\|^{2}
$$

using (3.41) for the last step. This controls the right-hand side of (5.2) by initial data, and indeed we see that if $\nabla \cdot \mathbf{b}_{\text {in }}=0$ then the discrete divergence $\nabla \cdot \mathbf{b}^{n}$ vanishes on each time step.

Now consider the velocity. Writing $p_{\mathrm{S}}^{n}=p_{\mathrm{S}}\left(\mathbf{u}^{n}\right)$, dotting (3.9) with $\nabla \phi$ we have

$$
\left\langle\frac{\mathbf{u}^{n+1}-\mathbf{u}^{n}}{\Delta t}, \nabla \phi\right\rangle+\nu\left\langle\nabla p_{\mathrm{S}}^{n}, \nabla \phi\right\rangle=\nu\left\langle\nabla\left(\nabla \cdot \mathbf{u}^{n+1}\right), \nabla \phi\right\rangle-\nu\left\langle\nabla \times\left(\nabla \times \mathbf{u}^{n+1}\right), \nabla \phi\right\rangle .
$$

Using the fact that

$$
-\nu\left\langle\nabla \times\left(\nabla \times \mathbf{u}^{n+1}\right), \nabla \phi\right\rangle=\nu\left\langle\nabla p_{\mathrm{S}}^{n+1}, \nabla \phi\right\rangle,
$$

one has

$$
\left\langle\frac{\nabla \cdot \mathbf{u}^{n+1}-\nabla \cdot \mathbf{u}^{n}}{\Delta t}, \phi\right\rangle+\nu\left\langle\nabla\left(\nabla \cdot \mathbf{u}^{n+1}+p_{\mathrm{S}}^{n+1}-p_{\mathrm{S}}^{n}\right), \nabla \phi\right\rangle=0 .
$$

As in [11], we let $q^{n}=\mathcal{Q} \mathbf{u}^{n}$ be the mean-zero solution of

$$
-\Delta q^{n}=\nabla \cdot \mathbf{u}^{n}, \quad \mathbf{n} \cdot \nabla q^{n}=0 \text { on } \Gamma .
$$

Note that $\left\|\nabla q^{n}\right\|$ is equivalent to $\left\|\nabla \cdot \mathbf{u}^{n}\right\|_{H^{1}(\Omega)^{\prime}}$ (here $H^{1}(\Omega)^{\prime}$ is the dual of $H^{1}(\Omega)$ ). Taking $\phi=q^{n+1}$ in (5.5), we find

$$
\left\langle\frac{\nabla q^{n+1}-\nabla q^{n}}{\Delta t}, \nabla q^{n+1}\right\rangle+\nu\left\langle\nabla \cdot \mathbf{u}^{n+1}+p_{\mathrm{S}}^{n+1}-p_{\mathrm{S}}^{n}, \nabla \cdot \mathbf{u}^{n+1}\right\rangle=0,
$$

whence

$$
\frac{\left\|\nabla q^{n+1}\right\|^{2}-\left\|\nabla q^{n}\right\|^{2}}{\Delta t}+\nu\left\|\nabla \cdot \mathbf{u}^{n+1}\right\|^{2} \leq \nu\left\|p_{\mathrm{S}}^{n+1}-p_{\mathrm{S}}^{n}\right\|^{2}
$$

and

$$
\left\|\nabla q^{n}\right\|^{2}+\nu \sum_{k=0}^{n-1}\left\|\nabla \cdot \mathbf{u}^{k+1}\right\|^{2} \Delta t \leq\left\|\nabla q^{0}\right\|^{2}+\nu \sum_{k=0}^{n-1}\left\|p_{\mathrm{S}}^{k+1}-p_{\mathrm{S}}^{k}\right\|^{2} \Delta t
$$


Using Lemma 8.1 of [11], we have the bound

$$
\left\|p_{\mathrm{S}}^{n+1}-p_{\mathrm{S}}^{n}\right\|^{2} \leq C\left\|\mathbf{u}^{n+1}-\mathbf{u}^{n}\right\|^{1 / 2}\left\|\mathbf{u}^{n+1}-\mathbf{u}^{n}\right\|_{H^{2}}^{3 / 2} .
$$

By Hölder's inequality and the stability estimate in Theorem 3.3,

$$
\begin{aligned}
& \sum_{k=0}^{n-1}\left\|p_{\mathrm{S}}^{k+1}-p_{\mathrm{S}}^{k}\right\|^{2} \Delta t \\
\leq & C\left[\sum_{k=0}^{n-1}\left\|\mathbf{u}^{k+1}-\mathbf{u}^{k}\right\|^{2} \Delta t\right]^{\frac{1}{4}}\left[\sum_{k=0}^{n-1}\left\|\mathbf{u}^{k+1}-\mathbf{u}^{k}\right\|_{H^{2}}^{2} \Delta t\right]^{\frac{3}{4}} \\
\leq & C \sqrt{\Delta t} .
\end{aligned}
$$

To control this by data, note that $\nabla q^{0}=(I-\mathcal{P}) \mathbf{u}^{0}$, hence as $\Delta t \rightarrow 0$,

$$
\left\|\nabla q^{0}\right\| \leq\left\|(I-\mathcal{P}) \mathbf{u}_{\text {in }}\right\|+\left\|\mathbf{u}^{0}-\mathbf{u}_{\text {in }}\right\|=\left\|(I-\mathcal{P}) \mathbf{u}_{\text {in }}\right\|+o(1) .
$$

Hence we find

$$
\left\|\nabla q^{n}\right\|^{2}+\nu \sum_{k=0}^{n-1}\left\|\nabla \cdot \mathbf{u}^{k+1}\right\|^{2} \Delta t \leq\left\|(I-\mathcal{P}) \mathbf{u}_{\mathrm{in}}\right\|+\left\|\mathbf{u}^{0}-\mathbf{u}_{\text {in }}\right\|+C \sqrt{\Delta t} .
$$

Acknowledgement. We thank Andy Majda for his help in originally facilitating our collaboration. This material is based upon work supported by the National Science Foundation under grant nos. DMS 06-04420 (RLP) and DMS 08-11177 (JGL), and partially supported by the Center for Nonlinear Analysis (CNA) under National Science Foundation Grant nos. 0405343 and 0635983.

\section{REFERENCES}

[1] R.A. Adams, Sobolev Spaces, Academic Press, San Diego, 1978.

[2] J.M. Ball, Strongly continuous semigroups, weak solutions, and the variation of constants formula, Proc. Amer. Math. Soc., 63, 370-373, 1977.

[3] R. Dautray and J.L. Lions, Mathematical Analysis and Numerical Methods for Science and Technology: Volume 3: Spectral Theory and Applications, Springer-Verlag, Berlin, 1990.

[4] G. Duvaut and J.L. Lions, Inéquations en thermoélasticité et magnétohydrodynamique, Arch. Rational Mech. Anal., 46, 241-279, 1972.

[5] L.C. Evans, Partial Differential Equations, American Mathematical Society, Providence, 1998.

[6] A. Friedman, Partial Differential Equations, Holt, Rinehart and Winston, New York, 1969.

[7] V. Georgescu, Some boundary value problems for differential forms on compact Riemannian manifolds, Ann. Mat. Pures Appl., 58, 159-198, 1979.

[8] V. Girault and P.A. Raviart, Finite Element Methods for Navier-Stokes Equations: Theory and Algorithms, Springer-Verlag, Berlin, 1986.

[9] J.D. Jackson, Classical Electrodynamics, Wiley, 3rd edition, 1998.

[10] O.A. Ladyzhenskaya, The Mathematical Theory of Viscous Incompressible Flow, Gordon and Breach, New York, 1969.

[11] J.G. Liu, J. Liu and R.L. Pego, Stability and convergence of efficient Navier-Stokes solvers via a commutator estimate, Comm. Pure Appl. Math., 60, 1443-1487, 2007.

[12] J.G. Liu, J. Liu and R.L. Pego, Error estimates for finite-element Navier-Stokes solvers without standard inf-sup conditions, Chinese Annals of Math., to appear.

[13] J.G. Liu, J. Liu and R.L. Pego, Stable and accurate pressure approximation for unsteady incompressible viscous flow, in preparation. 
[14] J.L. Lions and E. Magenes, Non-homogeneous Boundary Value Problems and Applications, Vol. I and II. Springer-Verlag, Berlin, 1972.

[15] M. Sermange and R. Temam, Some mathematical questions related to the MHD equations, Comm. Pure Appl. Math., 36, 635-664, 1983.

[16] L. Tartar, Topics in Nonlinear Analysis, Publications mathématiques d'Orsay 78.13. Université de Paris Sud, France, 1978.

[17] R. Temam, Navier-Stokes Equations: Theory and Numerical Analysis, AMS Chelsea, Providence, 2001. 